Review

\title{
Research Progress and Key Issues of Hydrodebenzylation of Hexabenzylhexaazaisowurtzitane (HBIW) in the Synthesis of High Energy Density Material Hexanitrohexaazaisowurtzitane (HNIW)
}

\author{
Xiaofei Tang ${ }^{1,+}$, Rui Zhu ${ }^{2, \dagger}$, Tianjing Shi ${ }^{2}$, Yu Wang ${ }^{2}$, Xiaochen Niu ${ }^{2}$, Yao Zhang ${ }^{1}$, Junchen Zhu ${ }^{1}$, Wei Li ${ }^{1}$, \\ Wanpeng $\mathrm{Hu}^{2, *}$ and Ruoqian $\mathrm{Xu}^{1, *}$ \\ $1 \quad$ Xi'an Modern Chemistry Research Institute, Xi'an 710065, China; tangxiaofei0807@163.com (X.T.); \\ 13488466218@163.com (Y.Z.); z18380461775@163.com (J.Z.); liubliw@163.com (W.L.) \\ 2 College of Biological, Chemical Sciences and Engineering, Jiaxing University, Jiaxing 314001, China; \\ z18324322682@163.com (R.Z.); stj19980802@163.com (T.S.); wangyu180310@163.com (Y.W.); \\ 88888888@zjxu.edu.cn (X.N.) \\ * Correspondence: hu688@zjxu.edu.cn (W.H.); nutar@163.com (R.X.) \\ + These two authors contributed equally to this work.
}

check for updates

Citation: Tang, X.; Zhu, R.; Shi, T.; Wang, Y.; Niu, X.; Zhang, Y.; Zhu, J.; Li, W.; Hu, W.; Xu, R. Research

Progress and Key Issues of

Hydrodebenzylation of

Hexabenzylhexaazaisowurtzitane (HBIW) in the Synthesis of High Energy Density Material

Hexanitrohexaazaisowurtzitane (HNIW). Materials 2022, 15, 409. https://doi.org/10.3390/ma15020409 Academic Editor: Jose Antonio Alonso

Received: 15 November 2021 Accepted: 29 December 2021 Published: 6 January 2022

Publisher's Note: MDPI stays neutral with regard to jurisdictional claims in published maps and institutional affiliations.

Copyright: (C) 2022 by the authors. Licensee MDPI, Basel, Switzerland. This article is an open access article distributed under the terms and conditions of the Creative Commons Attribution (CC BY) license (https:// creativecommons.org/licenses/by/ $4.0 /)$.

\begin{abstract}
High energy density materials (HEDM) are the subject of an extensive research effort in relation to the use of these compounds as components of rocket propellants, powders, and formulations of high-performance explosives. Hexanitrohexaazaisowurtzitane (HNIW, i.e., CL-20) has received much attention in these research fields for its specific impulse, burning rate, ballistics, and detonation velocity. In this paper, the development and performances of the explosives from the first to the fourth generation are briefly summarized, and the synthesis status of the fourth-generation explosive, HNIW, is reviewed. The key issues that restrict the development of industrial amplification synthesis of HNIW are analyzed, and the potential directions of development are proposed. It is pointed out that to synthesize new and efficient catalysts is the key to making the cost-effective manufacturing of CL-20 a reality.
\end{abstract}

Keywords: hexanitrohexaazaisowurtzitane (HNIW); hexabenzylhexaazaisowurtzitane (HBIW); hydrodebenzylation; catalyst development; catalyst deactivation

\section{The Development and Performances of the Explosives}

CL-20 (Hexanitrohexaazaisowurtzitane, HNIW) was firstly synthesized at the U.S. Naval Weapons Center in 1987 and became a major breakthrough in the field of high-energy materials. However, the history of explosives can be traced back to the mid-19th century [1].

When primary explosives, Mercury and Silver fulminates, had been discovered in Europe in the Middle Ages, a whole group of organic nitrates (nitro esters) was synthesized in the first half of the 19th century. For example, Nitrocellulose (pyroxylin) was obtained in 1846 by C. F. Schönbein. Approximately at the same time, Flores Demonte and Ménard received Mannitol hexanitrate (Nitromannite). Nitroglycerin (NG) was discovered by Ascanio Sobrero in 1847, whose molecular structure is shown in Figure 1. During the earlier stage after its discovery, the character of instability limited its application. Aliphatic nitro compounds were also synthesized around the middle of the 19th century. Tetranitromethane, for instance, was synthesized by the Russian chemist L. N. Shishkov in 1857. Trinitrotoluene (TNT), as shown in Figure 1, is a high-synthetic explosive, which was first prepared in 1863 by Julius Wilbrand. Due to its powerful explosive and stable nature, the compound played a huge role in World War II. It was used for the intensive shooting of artillery, and greatly increased the cruelty of the war. Aromatic nitro compounds, such as picric acid (2,4,6-trinitrophenol), were synthesized by Peter Woulfe for the first time using indigo during 1771. 
<smiles>O=[N+]([O-])OCC(CO[N+](=O)[O-])O[N+](=O)[O-]</smiles>

NG<smiles>Cc1c([N+](=O)[O-])cc([N+](=O)[O-])cc1[N+](=O)[O-]</smiles>

TNT<smiles>O=[N+]([O-])N1CN([N+](=O)[O-])CN([N+](=O)[O-])C1</smiles>

RDX<smiles>O=[N+]([O-])N1CN([N+](=O)[O-])CN([N+](=O)[O-])CN([N+](=O)[O-])C1</smiles>

HMX

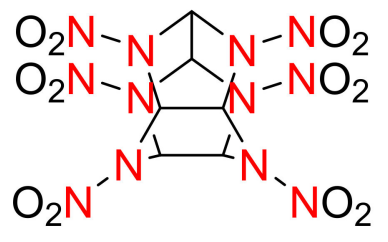

CL-20

Figure 1. Molecular structure of the I-IV-generation explosives.

Nitramine, i.e., $\mathrm{R} 1 \mathrm{R} 2 \mathrm{~N}-\mathrm{NO}_{2}$, is an extremely important type of energetic materials [2]. As shown in Figure 1, it mainly includes 1,3,5-trinitro-1,3,5-triazacyclohexane (RDX, found in 1899), and 1,3,5,7-tetranitro-1,3,5,7-tetraazacyclooctane (HMX, found in 1941), and was researched and synthesized after World War II, and had been the most comprehensively applied energetic material up to now. With a detonation velocity of $8500-8600 \mathrm{~m} / \mathrm{s}$, the RDX is perfectly suitable for large-scale suppression of multiple-rocket heavy guns, which can greatly improve the power and striking range of the weapon. The detonation velocity of HMX is $9000 \mathrm{~m} / \mathrm{s}$, which has a slightly higher impact sensitivity than TNT. It also has good comprehensive performances: it is stable and easier to be detonated. In the Gulf War, it was used for non-contact asymmetric warfare for long-range rocket missiles.

In the late 1970s, Yongzhong Yu, a professor at the Beijing Institute of Technology (BIT), and an expert on explosives in China's "Two bombs and One Star" project, focused on the molecular structure of explosive materials and proposed an innovative theory in which the structure was changed from a two-dimensional flat ring structure to a three-dimensional cage structure [3]. In 1979, Professor Yu and his collaborators successfully synthesized 4,10-dinitro-4,10-diazo-2,6,8,12-tetraoxyisowurtzitane $\left(5.5 .0 .0^{5,9} .0^{3,11}\right)\left(797^{\#}\right.$, as shown in Figure 2) [4,5], an explosive with a cage structure. They proposed that if the four oxygen atoms in the $797^{\#}$ molecule were replaced by four nitramine groups, the resulting compound, 2,4,6,8,10,12-hexanitro-2,4,6,8,10,12-hexaazaisowurtzitane $\left(5.5 .0 .0^{5,9} .0^{3,11}\right)$ (HNIW, known as CL-20), would have more comprehensive performances than HMX does, and would become a new-generation HEDM. As can be seen in Figure 2, two five-membered rings connecting four $\mathrm{N}-\mathrm{NO}_{2}$ groups and a six-membered ring connecting two $\mathrm{N}-\mathrm{NO}_{2}$ groups together make up the complicated molecular structure of HNIW, where the fist two rings are connected by a $\mathrm{C}-\mathrm{C}$ bond that has high tension in the polycyclic caged organic compound $[1,6]$. Professor Yu successfully synthesized this three-dimensional caged structure, CL-20, in 1994 [7]. Since then, a number of experts from BIT began to study the synthesis process of CL-20 [8]. It took 30 years for China to finally solve the key technology of industrial production, from constructing the three-dimensional cage-structure molecules explosive material proposed by Yu Yongzhong, to successful synthesis of $1 \mathrm{~kg}$ CL-20 samples using the "One-pot method" suggested by Yuxiang Ou, to the exploration and innovation of process route by Xinqi Zhao, and finally to the breakthrough of the industrial amplification key technology $[1,3,9]$. The detonation velocity of CL-20 is as high 
as $9500 \mathrm{~m} / \mathrm{s}$, the most powerful non-nuclear elemental explosive [1,3] with respect to being used in practical applications.
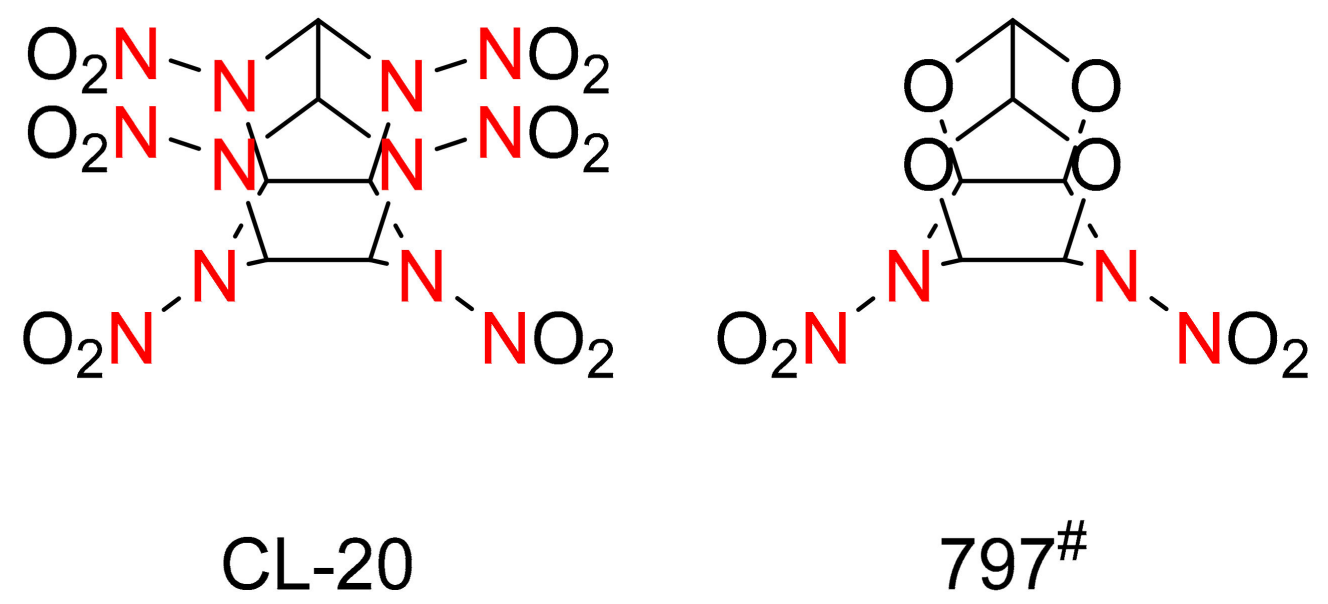

Figure 2. Molecular structures of CL-20 and 797\# [4].

American scholars proposed the idea of HNIW as a new generation of HEDM research in the early 1980s [9]. During that period of time, Nielsen synthesized HNIW in 1987, and published the synthesis route of CL-20 at the Fraunhofer Institute for Chemical Technology Annual Meeting in Germany in 1996 (see Figure 3). The developed HNIW synthesis route is divided into four processes: the first step is condensation, in which Benzylamine and glyoxal are condensed to hexabenzylhexaazaisowurtzitane (HBIW). The second step is debenzylation, where the six benzyl groups on HBIW are partially or completely converted to acetyl groups or other substituents to form the nitration precursor. The third is nitration, namely, the nitration precursor was nitrated into $\alpha$-CL-20 or $\gamma-C L-20$. The fourth is crystalstructure transformation, which stands for the conversion from $\alpha-C L-20$ or $\gamma-C L-20$ to $\varepsilon$-CL-20. This synthetic route has been widely used up to now. China and the United States independently completed the synthesis of CL-20 on the premise of mutual confidentiality from each other [1].<smiles></smiles>

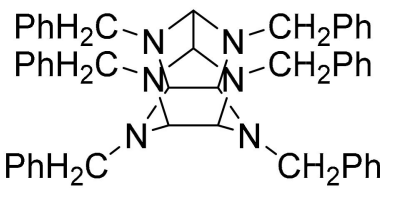

HBIW

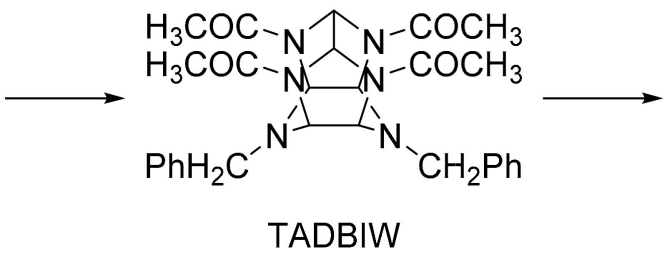

Figure 3. The synthesis route for CL-20 proposed by Nielsen [10].

A comparison of the molecular structures of the fourth-generation explosives is shown in Figure 1, and the physicochemical properties and explosion characteristics of the structures are listed in Table 1 [1]. 
Table 1. Comparisons of the physicochemical properties and explosion characteristics of the II-IVgeneration explosives $[1,10]$.

\begin{tabular}{ccccc}
\hline ITEM & TNT & RDX & HMX & CL-20 \\
\hline Density $/ \mathrm{g} \cdot \mathrm{cm}^{-3}$ & 1.65 & 1.82 & 1.90 & 2.04 \\
Relative density $/ \mathrm{g} \cdot \mathrm{cm}^{-3}$ & 91.64 & 91.77 & 91.88 & - \\
Oxygen balance/\% & -74.0 & -21.6 & -21.6 & -10.95 \\
Standard enthalpy of & -45.4 & 92.6 & 104.8 & 416.28 \\
formation $/ \mathrm{kJ} \cdot \mathrm{mol}^{-1}$ & 6.9 & 8.6 & 9.0 & $9.6^{* *}$ \\
Detonation velocity $/ \mathrm{km} \cdot \mathrm{s}^{-1}$ & 19 & 34 & 39 & $43^{* *}$ \\
Detonation pressure $/ \mathrm{GPa}$ & 738 & 903 & 886 & $827^{* *}$ \\
Critical capacity $/ \mathrm{cm}^{3} \cdot \mathrm{g}^{-1}$ & 353 & 120 & 720 & 54 \\
Friction sensitivity $/ \mathrm{N}$ & 15 & 7.4 & 287 & 4 \\
Impact sensitivity $/ \mathrm{N} \cdot \mathrm{m}$ & 300 & 230 & 228
\end{tabular}

* The smaller the value, the higher the friction sensitivity; ${ }^{* *}$ Calculated value.

As can be seen from Table 1, compared with HMX, the "king" of explosives, CL-20 has the advantages of higher energy density, lower oxygen balance, and higher enthalpy of formation $[3,7,11]$. Therefore, CL-20 has greater potential in applications [12]. For example, it can be used as the most powerful futuristic propellants or explosives due to the higher detonation velocity and detonation pressure. On the other hand, it has the disadvantages of poor sensitivity and low security. These drawbacks have to be fixed before being put into applications.

\section{Synthesis of HNIW}

A great deal of work has been done on the synthesis of CL-20 internationally, concentrating mainly on the following two aspects: the optimization and engineering amplification of the traditional synthesis route proposed by Nielsen, as shown in Figure 3, and the exploration of the new synthesis route of CL-20.

\subsection{Typical Synthesis Method}

Currently, researchers primarily use the route developed by Nielsen to synthesize HNIW (shown in Figure 3); that is, benzylamine (or substituted benzyl) and glyoxal were used as raw materials to produce HBIW through a condensation reaction. HBIW is extremely unstable in nitration medium, which leads to its cage structure opening rapidly, and thus, HNIW cannot be prepared by directly converting the benzyl group (substituted benzyl group) on HBIW to a nitro group. It is obliged to first convert part or all of the benzyl group into other functional groups that must have good stability to this cage structure and that are easy to be converted by the nitro group. Thus, the key step in HNIW synthesis is the debenzylation of HBIW; therefore, the current work introduces the debenzylation process of HBIW in detail [12].

In the HBIW debenzylation process, part or all of the benzyl groups are replaced by other functional groups (such as $\mathrm{CH}_{3} \mathrm{O}_{-}, \mathrm{C}_{2} \mathrm{H}_{5-}, \mathrm{CHO}-$ ) [13]. There are more than 15 nitrification precursors for the synthesis of CL-20 [14,15]. Up to now, only three nitration precursors have been manufactured at the kilogram level, which, respectively, are tetraacetyldibenzylhex-aazaisowurtzitane (TADBIW), tetraacetyldif-ormylhexazoisowoody (TADFIW), and tetraacetylhexazoisowoody alkane (TAIW). The TADBIW system is synthesized from HBIW by hydrodebenzylation once, and the latter two are synthesized from HBIW by hydrodebenzylation twice.

\subsubsection{Synthesis of TADBIW}

The most challenging work in synthesizing CL-20 is to convert HBIW to TADBIW (shown in Figure 4), which is caused not only by the multiplex chemical reaction program of HBIW, but also by its unique characteristics [16]. This process is simultaneous debenzylation-acetylation, where the $\mathrm{C}-\mathrm{N}$ bonds are broken down on the catalysts, which, 
in turn, causes the forming amines to acetylate with acetic anhydride [16]. Usually, HBIW, dimethylformamide, bromide, and a catalyst containing $\mathrm{Pd}(\mathrm{OH})_{2}$ are added to the reactor, and acetic anhydride follows after the air in the reactor is replaced with nitrogen. Then hydrogen is introduced into the reaction under certain conditions. After the reaction is completed, the purified TADBIW is obtained after being washed by solvent. One method to debenzylation has attained the highest level of popularity in the field, in which $\mathrm{H}_{2}$ and heterogeneous Pd-based catalysts are implemented in great amounts under catalytic reduction conditions [16]. Additionally, it is crucial to eliminate the benzyl groups under the conditions with the temperature ranges of $30^{\circ} \mathrm{C}$ to $60^{\circ} \mathrm{C}$, and with acetic anhydride as an acylation reagent [16]. The complex cage structure is the main factor contributing to the disadvantage that HBIW is unstable and easy to decompose when heated [16]. In order to maintain the cage structure, it is essential to launch the debenzylation and to transform it in a short time at a low temperature (generally $15-23^{\circ} \mathrm{C}$ ). It has been proved by plenty of optimization experiments that the better starting temperature falls in the range of $17-19^{\circ} \mathrm{C}$. Thus, in the above-mentioned reaction process, the catalysts play an important role when providing high efficiency at around room temperature [12].

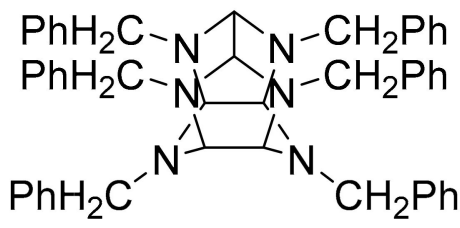

HBIW
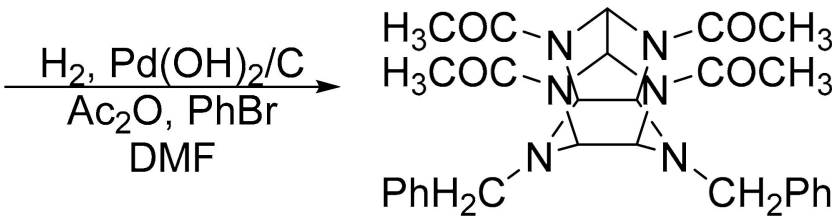

TADBIW

Figure 4. Synthesis of TADBIW.

Catalyst development. It has taken several decades for researchers to develop Pd/C catalysts as the key for the hydrogenation debenzylation of HBIW [6]. In 1997, Nielsen and Barbara succeeded in synthesizing TADBIW, and they only used $20 \mathrm{wt}$.\% Pearlman's $\mathrm{Pd} / \mathrm{C}$ catalyst and nearly the same amounts of HBIW. Based on their works, scientists have investigated numerous $\mathrm{Pd} / \mathrm{C}$ catalysts for commerce, which have significantly increased the catalytic activity [6]. In 1997, Wardle revealed that Degussa E101 NE/W had yields reaching $90 \%$ for hydrogenating HBIW when keeping the scale in a 50-g process, and with help from bromobenzene. Additionally, the material is recycled once more. Using different carbon precursors as catalysts supports, and subunit-supported 6 or $10 \mathrm{wt}$ \% Pd had a yield as high as $82 \%$ when keeping the experiment scale in a 3-g process [17]. It was reported that in the chemical process, ACROS organic active carbon over a Pd catalyst performed better than other types of activated carbons [17]. Disordered mesoporous carbon (MC) had a yield of TADBIW as high as $80 \%$, a very good catalytic reactivity, when it was prepared using the hard template method [6], while the yield of the prepared $\mathrm{Pd}(\mathrm{OH})_{2} / \mathrm{MC}$ catalyst, as a comparison, remained above $70 \%$ after two cycles. The researchers found that it is hard to obtain the target product when loading Pd with a concentration lower than $5 \mathrm{wt}$.\% if the usage amount of the catalysts remains constant [17].

Effect factors. It has been revealed by researchers that the catalytic activity of $\mathrm{Pd}$ $(\mathrm{OH})_{2} / \mathrm{C}$ is significantly affected by the following factors [18-21], such as the surface physical properties of activated carbon, reaction temperature, and the loading amount of $\mathrm{Pd}$, etc. It has also been revealed that lower temperatures make $\mathrm{Pd}(\mathrm{OH})_{2}$ particles disperse better. In the $20-\mathrm{kg}$ scale, a series of amplification trials has implemented the catalyst for the hydrogen debenzylation from HBIW, and the results showed that the yield of the TADBIW product reached as high as $82 \%$.

Effects of additives containing bromine. In 1995, Wardle and Edwards [22] found that the addition of bromide made a cost-effective way of synthesizing HNIW. When $\mathrm{Pd}(\mathrm{OH})_{2}$ was used as catalyst without support, a small amount of bromobenzene could help receive excellent effects, such as shorter reaction times, less catalyst amounts, and 
higher yields of TADBIW (from $68.3 \%$ to $80 \%$ ). Weirong Han and Yuxiang Ou et al. [21] also drew a similar conclusion after investigating the effects of 10 types of bromides, including bromobenzene, imposing on HBIW catalytic hydrodebenzylation, and discussed the mechanisms. Lianzhong Chen and Xinqi Zhao et al. [23] found that switching bromobenzene to bromotoluene $(\mathrm{o}, \mathrm{m}, \mathrm{p}$-mixture) in the primary hydrodebenzylation of HBIW improved the hydrodebenzylation yield, and reduced the amount and toxicity of bromine-source substances. In addition, Weirong Han [24,25] and Yuxiang Ou et al. [26,27] prepared triacetyltribenzylhexazazoisovudane (TATBIW), an important intermediate in the hydrolytic reaction, analyzed its single-crystal structure (TATBIW $\cdot 0.5 \mathrm{H}_{2} \mathrm{O}$ ) [28], and discussed the mechanism and reaction kinetics of the reaction.

Process development. Professor Yongzhong Yu, respectively, synthesized TADBIW in 1993 and CL-20 [4] in June 1994, which coincides with the later-reported methods proposed by Nielsen, France, etc. In 1995, Bellamy published a research report [29] on the preparation of TADBIW from HBIW by hydrodebenzylation-acetylation, pointing out that the yield of the product had been improved by appropriately increasing the reaction temperature, prolonging the reaction time, or increasing the amount of catalyst, i.e., $\mathrm{Pd}(\mathrm{OH})_{2} / \mathrm{C}$. It concluded that direct nitration TADBIW produced high-yield CL-20, but the specific nitration medium and related process conditions were not provided [6,29]. Since TADBIW can be obtained by hydrodebenzylation only once, many countries and institutes initially adopted the one-pot nitration route for the synthesis of CL-20 [30-33], which is easy to realize at the laboratory scale. Some countries, such as the United States and France, have found that this method is not suitable for engineering amplification [34,35], because it takes a large amount of noble metals and is easy to inactivate, resulting in a high cost [36], while the existing economical catalysts are not recyclable, limiting their mass production and comprehensive application [16]. However, other countries, such as Iran and Russia, are still continuing the relevant research [37-40].

\subsubsection{Synthesis of TADFIW}

As shown in Figure 5, the synthesis of TADFIW is based on TADBIW. Add TADBIW and formic acid into a reaction bottle, remove the air in the reaction bottle, and introduce normal-pressure hydrogen at a certain temperature for hydrogenolysis. The reaction stops when the system no longer absorbs hydrogen. After the reaction is finished, the TADFIW with certain purity can be obtained after the operations of washing, decompression, and the like. In 1997, Thiokol published the detailed process and conditions for the synthesis of HNIW by using TADFIW as a nitration precursor. In this process, bromobenzene was used as the bromine source and dimethylformamide (DMF) as the solvent [22,41]. DMF is a Lewis base, which can neutralize the acid generated in the medium or in the reaction process, keep the medium in a low-acidic environment, increase the solubility of HBIW in the hydrolytic medium, accelerate the reaction speed of hydrolytic acetylation, reduce the existence time of HBIW in the reaction medium, prevent and halt the breakdown of the cage structure, and reduce the decomposition of the reaction substrate. The yield of TADBIW reached more than $80 \%$ by Thiokol, and it was further hydrolyzed in $\mathrm{HCOOH}$ medium to obtain TADFIW. TADFIW was then nitrated in nitric-sulfuric mixed acids to form the target product, CL-20, with a high yield. The United States used the TADFIW route to prepare CL-20 up to ten tons. However, formyl acetyl was difficult to be separated, which resulted in the CL-20 containing 2\% 3\% of pentanitromonoformylhexazazoisowoodane impurity, which affected the detonation and the safety performance of CL-20 [3,42,43]. After 2002, the United States abandoned the TADFIW route; however, some countries, such as Iran, are still working on the relevant work [20]. 


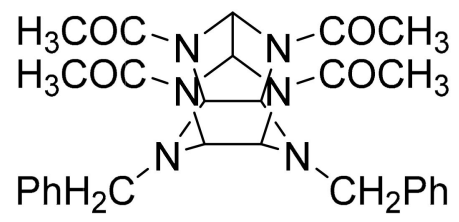

TADBIW

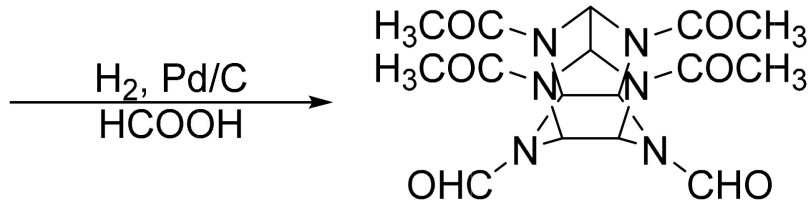

TADFIW

Figure 5. Synthesis of TADFIW.

\subsubsection{Synthesis of TAIW}

HAIW is an excellent intermediate for the synthesis of HNIW. The synthetic route is as follows: TADBIW is placed in a mixed solution of methanol and formic acid to form a slurry. Add $\mathrm{Pd} / \mathrm{C}$ catalyst into that slurry under stirring, heat it for a certain time, and then filter and extract it to separate the TAIW from it. This route was pioneered in China. In 1994, Xinqi Zhao hydrogenated TADBIW in acetic acid medium to prepare TAIW, and then synthesized CL-20 from TAIW [44]. Cai Wang and Yuxiang Ou et al. [45,46] studied and reported the crystal structure of HAIW. As shown in Figure 6, Jinquan Liu and Yuxiang Ou et al. used propionic acid and n-butyric acid as hydrogenolysis media, and a self-made Pd substrate as a catalyst to hydrolyze TADBIW for producing TAIW [47]. The hydrodebenzylation of TADBIW in $\mathrm{HCOOH} / \mathrm{CH}_{3} \mathrm{OH}$ medium for obtaining HAIW was studied by Thiokol [22,41].

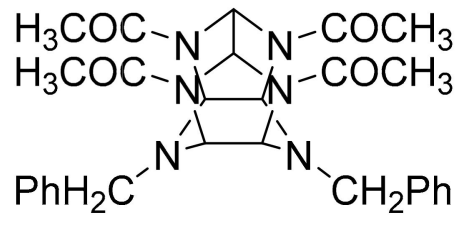

TADBIW

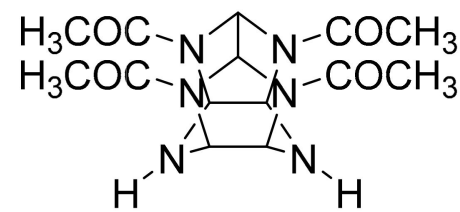

TAIW

Figure 6. Synthesis of TAIW.

As the intermediate production, TADBIW showed higher performance than HBIW in terms of stability and durability at high temperatures. To accelerate the process of the debenzylation of TADBIW, the heating can be maintained at higher temperatures (usually $34-40^{\circ} \mathrm{C}$ ) [6]. It is difficult for TADBIW to solutate in other alternative solvents; its debenzylation must be conducted in formic or acetic acid solution. However, the catalysts in the acid solvents can be etched by the solution, resulting in poor cycle catalytic properties, which has been reported. There is a declining trend in the catalytic performances for the hydrogenation of N,N-dibenzyl-3-phenylindolizin-1-amine when it is in the acetic acid solution with cycles over recycled $\mathrm{PtO}_{2}$ and $\mathrm{Pt} / \mathrm{C}$ [12]. According to the report, the catalysts must be stable so that they can resist the acid corrosion. The Pd/C with $10 \%$ loading is the most common material in the field for the hydrodebenzylation of HBIW and TADBIW. However, the low atom-utilization rate, as well as the loss and aggregation of Pd, restrain the catalytic performance. In the field of HEDMs, it is a significant work to develop a new catalyst that has high effectiveness for the debenzylation of both HBIW and TADBIW [12].

At present, most countries have adopted the TAIW route for synthesizing CL-20 [37,48,49]. The nitration of TAIW for producing high-purity CL-20 has been achieved smoothly by using industrial nitration agents in standard nitration units [43]. The TAIW route requires noble metal Pd-based catalysts for hydrodebenzylation twice; thus, the preparation of an efficient $\mathrm{Pd}$ catalyst and the recycling of $\mathrm{Pd}$ catalysts are the key technologies to make CL-20 for wide use [7,12]. 


\subsection{Other Synthesis Methods}

\subsubsection{Non-Hydrodebenzylation-Oxidation Debenzylation of HBIW}

Due to the long process route of HBIW hydrodebenzylation and nitration, and the massive requirement of noble metal catalysts in the hydro removal of benzyl groups, the manufacture of CL-20 costs huge sums of investment in reality. In order to reduce the cost and simplify the synthesis procedures of CL-20, researchers all over the world are trying to develop new synthetic routes [7].

HBIW reacts with trimethylsilane ethyl chloroformate, creating the corresponding substituted products in solvent-mixed tetrahydrofuran and ethyl ether, which has been revealed by the Asahi Kasei Corporation. This reaction requires chloroformate-substituted products which can be generated in an inert gas environment, and CL-20, which is obtained when there is nitrite and nitric acid. Because chloroformate is highly toxic and expensive, the synthesis cost of CL-20 by this method is much higher than the hydrodebenzylation method; therefore, this method has not been applied in practice. Surapaneni et al. [50], from the US Army Research and Development Center, failed to achieve the hydrogenfree debenzylation of HBIW using strong Lewis acid, because HBIW decomposed easily under the reaction conditions. Siping Pang and Yongzhong Yu from BIT studied the oxidation debenzylation reaction of HBIW or HADBIW [51-62], and made the oxidation debenzylation manufacturing of CL-20 a reality, using $\mathrm{KMnO}_{4}$ and ammonium nitrate (IV) as oxidants, $\mathrm{CH}_{2} \mathrm{Cl}_{2}$ and DMF as solvents, $\mathrm{Ac}_{2} \mathrm{O}$ and nitric acid (65\%)/ sodium nitrite as reactants, and the combination of boron trifluoride ether, ammonium persulfate, sodium nitrite, anhydrous sodium carbonate, and tetraethylammonium bromide as catalysts. Since then, similar studies have been carried out in India and other countries [63]. Due to plenty side-reactions, the yield and purity of the products by this method cannot meet the requirements of engineering production, so it does not have practical application [7]. The bottleneck of a hydrogen-free solution lies in the poor stability of HBIW in the reaction medium, and the reaction activity of the six benzyl groups in HBIW is different, which is difficult to be completely converted at once [7].

\subsubsection{Non-HBIW Route-Synthesis of Other Iso-Woodsane Precursors}

The non-HBIW route attempts to synthesize other iso-woodsane precursors and then direct nitrate into CL-20, aiming to shorten the synthesis route and reduce the cost. Sysolyatin et al. [64], from Russia, reported the condensation of sulfamate with glyoxal to obtain the caged precursor of isodurane and the product nitrated into CL-20. In 2005, Cagnon et al., from a French dynamite company [65], used aromatic heterocyclic methylamine or allylamine with acetate glyoxal to synthesize hexaazazoisovaltane, which was then directly nitrated to obtain CL-20. On the basis of the above-mentioned methods, Chapman et al. [66,67], from the United States, used photocatalytic and oxidation methods to make the isomerization of hexaallyl hexaazazozygrotane, and nitrated the product to CL-20. Related studies have also been carried out in Poland and other countries, but the researchers did not make much substantial progress. The researchers from BIT $[68,69]$ also synthesized some new isowurtzitane derivatives. Moreover, Anxin Hou, from the Southeast University of China [70], synthesized a cage precursor, hexafurfurylhexaazaisowurtzitane (HFIW), and directly nitrated the HFIW into HNIW. All above works have the problems of low yield of HNIW and no breakthrough in the practical application of engineering.

2.2.3. High-Energy Materials with Non-Cage Structures-Three-Dimensional MOFs with Nitrogen-Rich Elements

In order to resolve the contradiction between high energy and high sensitivity in the development of energy materials, Siping Pang et al., from BIT [1], designed and synthesized nitrogen-rich three-dimensional MOFs. The presence of nitrogen-containing heterocycle ligands guarantees the synthesized material with high energy density, while the sensitive nitrogen and metal atoms are clad in the three-dimensional structure, which ensures the decrease of sensitivity and the improvement of safety. 
The above new synthesis technologies of CL-20 have encountered the bottleneck of low yields and side-reactions, and the characteristics of the cage structure of isodurane was unstable and easy to be destroyed, which makes the mild catalytic hydrodebenzylationbe an inevitable choice. To break through the bottleneck, there are needs for new methods, or new technologies. However, it is not a short-term work; thus, the HBIW hydrodebenzylation route is still a feasible means for mass production [7].

\section{Problems in HNIW Engineering Manufacturing}

Many countries have carried out a lot of works on the synthesis and manufacture of CL-20. Although the engineered CL-20 has been made into a reality, and though the cost has been gradually reduced, it is still relatively high compared to the third-generation energetic compounds RDX and HMX. Practices have proved that only the hydrogenolysis debenzylation method, using precious metal $\mathrm{Pd}$ as a catalyst, has practical significance.

At present, researchers have recognized [18-21] that a carbon-supported Pd catalyst is the most effective catalyst for HBIW hydrodebenzylation reaction, and the amount of Pd is about $20 \mathrm{wt}$ \% of the supporting carbon-based material. When the amount of Pd loaded on the catalyst was $0.3 \mathrm{wt}$.\% of the substrate HBIW, the hydrogenolysis debenzylation acetylation product yielded up to 93\%. Weirong Han and Yuxiang Ou et al. [71] found that the yield of TADBIW could reach $88 \%$ when the amount of Pd was $0.2 \mathrm{wt}$.\% of HBIW. The carbon-based material-supported Pd catalysts have high conversion rates and selectivity, but the reaction activity of the Pd-catalyzed hydrodebenzylation process is unstable; sometimes the catalytic effect significantly declined, and even catalyst poisoning can occur, which results in hydrodebenzylation failure and the difficulty to reuse catalysts [65]. All the above reasons make HNIW synthesis price too high, and is the biggest obstacle to mass production of the energetic material HNIW.

Wardle [22] found that N-benzylacetamide, the by-product in the HBIW hydrodebenzylation process, led to Pd catalyst poisoning, and he also found that the generation of benzylamine was controlled by regulating the temperature of the hydrodebenzylation reaction. However, it is inevitable to contain a certain amount of N-benzylacetamide in HBIW during its mass-production process. Its purity can reach higher than $98 \%$ after being refined through recrystallization, but it still contains a small amount of impure oxalodibenzylamine produced in the oxidation of aldehyde amine condensation intermediates, $\mathrm{C}_{6} \mathrm{H}_{5} \mathrm{CH}_{2} \mathrm{NHCH}(\mathrm{OH}) \mathrm{CH}(\mathrm{OH}) \mathrm{NHCH}_{2} \mathrm{C}_{6} \mathrm{H}_{5}$, and affects the performance of the Pd-based catalyst during the hydrodebenzylation process [72,73]. I. L. Simakova et al. [39] discovered that the rapid deactivation of a Pd/C catalyst in the HBIW hydrodebenzylation process was not determined by the preparation method, but the agglomeration of metal particles and the blocking of activated carbon particles by oligomer products inside the carbon pores. Hadis Bashiri et al. [74] found that the loading amount of Pd decreased in the HBIW hydrodebenzylation process, and believed that the leaching and aggregation of $\mathrm{Pd}$ particles was responsible for the deactivation of the catalyst. To reduce the loading amount of Pd and improve the stability of the Pd-based catalyst, JunYang and Shuang Liu, from the Shanghai Institute of Organic Chemistry, Chinese Academy of Sciences, studied [11,12,16] the application of bimetallic catalysts in HBIW hydrodebenzylation reactions, and found that when the amount of the catalyst was the same, the yield of a bimetallic catalyst (PdFe) was significantly higher than that of the Pd mono-metal catalyst, and was $4 \%$ higher than that of the commercial Pd/C catalyst.

Although there have been reports on the deactivation reasons for HBIW hydrodebenzylation reaction carbon-supported Pd catalysts and the design and development of new catalysts $[1,6]$, these studies are rarely systematic and are not published. In order to save the cost and further reduce the consumption of raw materials, in-depth research on the causes and mechanisms of catalyst deactivation play a great guiding role in the optimization of the catalyst structure, the construction of new catalyst structures, and the improvement of reaction processes. In the HBIW hydrodebenzylation process, under the precondition of maintaining the catalytic activity and selectivity of HBIW for hydrodebenzylation reaction, 
seeking cheap and efficient new catalysts, reducing the use of palladium, or prolonging the life of Pd-based catalysts are the development directions for eventually making the large-scale production and application of HNIW a reality.

Author Contributions: Writing—original draft preparation, X.T., R.Z., T.S., Y.W., X.N., Y.Z., J.Z. and W.L.; writing-review and editing, W.H. and R.X.; supervision, W.H. and R.X.; project administration, W.H. and R.X.; funding acquisition, X.T. and R.X. All authors have read and agreed to the published version of the manuscript.

Funding: This research received no external funding.

Institutional Review Board Statement: Not applicable.

Informed Consent Statement: Not applicable.

Data Availability Statement: Not applicable.

Conflicts of Interest: The authors declare no conflict of interest.

$\begin{array}{ll}\text { Nomenclature } \\ \text { HBIW } & \text { hexabenzylhexaazaisowurtzitane } \\ \text { HFIW } & \text { hexafurfurylhexaazaisowurtzitane } \\ \text { HAIW } & \text { hexaacetylhexaazaisowurtzitane } \\ \text { HEDM } & \text { high energy density materials } \\ \text { HNIW } & \text { hexanitrohexaazaisowurtzitane } \\ \text { TADBIW } & \text { tetraacetyldibenzylhex-aazaisowurtzitane } \\ \text { TADFIW } & \text { tetraacetyldif-ormylhexazoisowoody } \\ \text { TAIW } & \text { tetraacetylhexazoisowoody } \\ \text { NG } & \text { nitroglycerine } \\ \text { TNT } & \text { trinitrotoluene } \\ \text { RDX } & \text { 1,3,5-trinitroperhydro-1,3,5-triazine } \\ \text { HMX } & \text { octahydro-1,3,5,7-tetranitro-1,3,5,7-tetrazocine }\end{array}$

\section{References}

1. Qing, L. Study on the Morphology and Particle Size Control of CL-20; Beijing Institute of Technology: Beijing, China, 2016.

2. Bayat, Y.; Malmir, S.; Hajighasemali, F.; Dehghani, H. Reductive Debenzylation of Hexabenzylhexaazaisowurtzitane using Multi-walled Carbon Nanotube-supported Palladium Catalysts: An Optimization Approach. Cent. Eur. J. Energ. Mat. 2015, 12, 439-458.

3. Explosives Subject of BIT: Aspiring to the Highest Peak of Explosives in the World. Available online: http:/ / edu.sina.com.cn/ gaokao/2016-04-19/doc-ifxriqqx3013740.shtml (accessed on 19 April 2016).

4. Yu, Y.; Guan, X. Studies on the Synthesis of Hexanitrohexaazaisowurtzitane. Chin. J. Energ. Mater. 1999, 7, 1-4.

5. Chen, F.; Duan, B.; Yu, Y. Research Reports Compilation of Chinese Ministry of Armament Industry; No. 214. Research Institute (1978-1980); Chinese Ministry of Armament Industry: Bengbu, China, 1983.

6. Zhang, M.; Liu, S.; Li, L.; Li, X.; Huang, H.; Yin, J.; Shao, X.; Yang, J. Effect of Carbon Supports on Pd Catalyst for Hydrogenation Debenzylation of Hexabenzylhexaazaisowurtzitane (HBIW). J. Energ. Mater. 2017, 35, 251-264. [CrossRef]

7. Pang, S.; Shen, F.; Lyu, P.; Dong, K.; Zhang, Y.; Sun, C.; Song, J.; Zhao, X. Research Progress in Synthesis of Hexanitrohexaazaisowurtzitane. Acta Armamentarii 2014, 35, 725-732.

8. Ou, Y.; Chen, B.; Jia, H.; Pan, Z.; Xu, Y. Structural Identification of Hexanitrohexaazaisowurtzitane. Chin. J. Energ. Mater. 1995, 3, $1-8$.

9. Nielsen, A.T. Synthesis of caged nitramine explosives. In Proceedings of the JANNAF Joint Army Navy NASA Air Force Propulsion Meeting, San Diego, CA, USA, 15-17 December 1987.

10. Maksimowski, P.; Golofit, T.; Tomaszewski, W. Palladium Catalyst in the HBIW Hydrodebenzylation Reaction. Deactivation and Spent Catalyst Regeneration Procedure. Cent. Eur. J. Energ. Mat. 2016, 13, 333-348. [CrossRef]

11. Yang, J.; Liu, S. Application of Palladium Bimetallic Catalyst in HBIW Catalytic Hydrolysis. China Patent CN 106,946,894 A, 14 July 2017.

12. Lou, D.; Wang, H.; Liu, S.; Li, L.; Zhao, W.; Chen, X.; Wang, J.; Li, X.; Wu, P.; Yang, J. PdFe bimetallic catalysts for debenzylation of hexabenzylhexaazaisowurtzitane (HBIW) and tetraacetyldibenzylhexaazaisowurtzitane (TADBIW). Catal. Commun. 2018, 109, 28-32. [CrossRef]

13. Ou, Y.; Xu, Y.; Chen, J.; Chen, B.; Zheng, F.; Jia, H.; Wang, C. Synthesis of Cage Heterocyclic Compounds with High Strain. Chem. J. Chin. Univ. 1999, 20, 561-564. 
14. Gong, X.; Sun, C.; Pang, S.; Zhang, J.; Li, Y.; Zhao, X. Research Progress in Study of Isowurtzitane Derivatives. Chin. J. Org. Chem. 2012, 32, 486-496. [CrossRef]

15. Ou, Y.; Liu, J.; Wang, Y.; Meng, Z. Synthesis and Industrial Production of Five Hydrogenolysis-debenzylation Compounds from Hexabenzylhexaazaisowurtzitane. Chin. J. Org. Chem. 2005, 68, 731-735.

16. Liu, S.; Ji, F.; Li, X.; Pan, X.; Chen, S.; Wang, X.; Zhang, Y.; Men, Y. Stick-like mesoporous titania loaded Pd as highly active and cost effective catalysts for hydrodebenzylation of hexabenzylhexaazaisowurtzitane (HBIW). Mol. Catal. 2019, 477, 110556. [CrossRef]

17. Zhang, M. Preparation and Performances of Palladium Carbon Catalysts Supported by Different Type Carbon Materials; Liaocheng University: Liaocheng, China, 2016.

18. Fotouhi-Far, F.; Bashiri, H.; Hamadanian, M.; Keshavarz, M.H. Increment of activity of $\mathrm{Pd}(\mathrm{OH})(2) / \mathrm{C}$ catalyst in order to improve the yield of high performance 2,4,6,8,10,12-hexanitrohexaazaisowurtzitane (HNIW). Inorg. Nano-Met. Chem. 2017, 47, 1489-1494. [CrossRef]

19. Qiu, W.; Jiang, J.; Sun, C.; Pang, S.; Liu, H.; Zi, X.; Zhang, G.; He, H. Preparation and characterization of highly active hydrodebenzyl $\mathrm{Pd}(\mathrm{OH}) 2 / \mathrm{C}$ catalyst. In Proceedings of the 9th Chinese National Annual Conference on Industrial Catalytic Technology and Application, Xiamen, China, 1 August 2012; pp. 197-199.

20. Bayat, Y.; Ebrahimi, H.; Fotouhi-Far, F. Optimization of Reductive Debenzylation of Hexabenzylhexaazaisowurtzitane (the Key Step for Synthesis of HNIW) Using Response Surface Methodology. Org. Process Res. Dev. 2012, 16, 1733-1738. [CrossRef]

21. Qiu, W.; Liu, H.; Dong, K.; Sun, C.; Pang, S.; Bai, G.; Zi, X.; Zhang, G.; He, H. Preparation of Pd(OH)2/C Catalyst for Hydrogenolytic Debenzylation of Hexabenzylhexaazaisowurtzitane. Chin. J. Energ. Mater. 2014, 22, 441-446.

22. Wardle, R.B.; Edwards, W.W. Hydrogenolysis of 2,4,6,8,10,12-Hexabenzyl-2,4,6,8,10,12-Hexaazatetracyclo [5.5.0.05,9.03,11] Dodecane: World Intellectual Property Organization. US Patent US 5,739,325 A, 14 April 1998.

23. Chen, L.; Fang, T.; Guo, X.; Zhao, X. Investigation on Hydrogrnolysis of Hexabenzylhexaazaisowurtzitane. Chin. J. Explos. Propell. 2002, 25, 29-30.

24. Han, W.; Ou, Y.; Zhang, X.; Huang, X.; Mou, W.; Gao, Y. Hydrogenolysis of Triacetyltribenzylhexaazaisowurtzitane and Tetraacetyldibenzylhexaazaisowurtzitane. Chin. J. Energ. Mater. 2008, 16, 153-155.

25. Liu, J.; Wang, J.; Han, W.; Lu, L. Study on Hydrogenolysis of HBIW and Crystal Structures of the Reaction Products. Chin. J. Energ. Mater. 2003, 11, 4-7.

26. Ou, Y.; Jia, H.; Chen, B.; Fan, G.; Xu, Y.; Pan, Z.; Wang, C. Synthesis and Crystal Structure of Hexanitrohexazaisowoodane. Sci. China Ser. B 1999, 1, 39-46.

27. Chen, J.; Zheng, F.; Ou, Y.; Chen, B.; Wang, Z.; Li, B. Study on the Kinetics of Hydrogenolysis Debenzylation of 2,4,6,8,10,12 Hexabenzyl 2,4,6,8,10, 12 Hexaazaisowurtzitane(HBIW). Chin. J. Beijing Inst. Technol. 1999, 9, 133-136.

28. Han, W.; Ou, Y.; Liu, J.; Chen, B. Synthesis and Crystal Structure of Triacetyltribenzyl-hexaazaisowurtzitane (TATBIW•0.5H2O). Chin. J. Org. Chem. 2005, 25, 114-119.

29. Bellamy, A. Reductive debenzylation of hexabenzylhexaazaisowurtzitane. Tetrahedron 1995, 51, 4711-4715. [CrossRef]

30. Duddu, R.; Dave, P.R. Process and Compositions for Nitration of N-Nitric Acid at Elevated Temperatures to Form HNIW and Recovery of Gamma HNIW with High Yields and Purities and Crystallizations to Recover Epsilon HNIW Crystals. US Patent US 6,160,113 A, 12 December 2000.

31. Simpson, R.L.; Lee, R.S.; Tillotson, T.M.; Hrubesh, L.W.; Swansiger, R.W.; Fox, G.A. Process for Preparing Energetic Materials. US Patent US 8,075,716 B1, 13 December 2011.

32. Lü, L.; Ou, Y.; Wang, J. Nitrosolysis-debenzylation to Tetraacetyldibenzylhexaazaisowurtzitane. Chin. J. Explos. Propell. 2003, 26, 41-43.

33. Lü, L.; Ou, Y.; Wang, J. Synthesis and Crystal Strucutre of Tetraacetyldinitroso-hexaazaisowurtzitane (TADNIW•H2O). Chin. J. Org. Chem. 2005, 4, 399-404.

34. Bayat, Y.; Hajimirsadeghi, S.S.; Pourmortazavi, S.M. Statistical Optimization of Reaction Parameters for the Synthesis of 2,4,6,8,10,12-Hexanitro-2,4,6,8,10,12-hexaazaisowurtzitane. Org. Process Res. Dev. 2011, 15, 810-816. [CrossRef]

35. Stierstorfer, J.; Klaptke, T.M. High Energy Materials. Propellants, Explosives and Pyrotechnics. By Jai Prakash Agrawal. Angew. Chem. Int. Edit. 2010, 36, 6253. [CrossRef]

36. Latypov, N.V.; Wellmar, U.; Goede, P.; Bellamy, A.J. Synthesis and Scale-Up of 2,4,6,8,10,12-Hexanitro-2,4,6,8,10,12hexaazaisowurtzitane from 2,6,8,12-Tetraacetyl-4,10-dibenzyl-2,4,6,8,10,12-hexaazaisowurtzitane (HNIW, CL-20). Org. Process Res. Dev. 2000, 4, 156-158. [CrossRef]

37. Bayat, Y.; Mokhtari, J. Preparation of 2,4,6,8,10,12-hexanitro-2,4,6,8,10,12-hexaazaisowurtzitane from 2,6,8,12-tetraacetyl 2,4,6,8,12hexaazaisowurtzitane using Various Nitrating Agents. Def. Sci. J. 2011, 61, 171-173.

38. Mandal, A.K.; Pant, C.S.; Kasar, S.M.; Soman, T. Process Optimization for Synthesis of CL-20. J. Energ. Mater. 2009, 27, 231-246. [CrossRef]

39. Koskin, A.P.; Simakova, I.L.; Parmon, V.N. Reductive debenzylation of hexabenzylhexaazaisowurtzitane-The key step of the synthesis of polycyclic nitramine hexanitrohexaazaisowurtzitane. Russ. Chem. Bull. 2007, 56, 2370-2375. [CrossRef]

40. Maksimowski, P.; Fabijańska, A.; Adamiak, J. Tetraaeetyl-dibenzyl-hexaazaisowurtzitane nitrosation-Studies on scale-up synthesis of HNIW. Propell. Explos. Pyrot. 2010, 35, 353-358. [CrossRef]

41. Bazaki, H.; Kawabe, S.; Miya, H.; Kodama, T. Synthesis and sensitivity of hexanitrohexaazaisowurzitane (HNIW). Propell. Explos. Pyrot. 1999, 23, 333-336. [CrossRef] 
42. Ou, Y.; Xu, Y.; Chen, B.; Liu, L.; Wang, C. Synthesis of Hexanitrohexaazaisowurtzitane from Tetraacetyldiformylhexaazaisowurtzitane. Chin. J. Org. Chem. 2000, 20, 556-559.

43. Zhao, X.; Fang, T.; Sun, C. Research and Development of HNIW synthesis. Acta Armamentarii 2004, $25,354-358$.

44. Zhao, X.; Shi, N. The Crystal Structure of $\varepsilon$-hexanitrohexazazozygrotane. Chin. J. Chem. 1995, 23, 2158-2160.

45. Wang, C.; Ou, Y.; Chen, B. Synthesis of Hexaacetylhexaazaisowurtzitane. Chin. J. Chem. World 2000, 9, 462-464.

46. Wang, C.; Ou, Y.; Chen, B. Synthesis and crystal structure of hexaacetylhexaazaisowurtzitane. Chin. J. Chem. Online 2000, 3, 44. [CrossRef]

47. Liu, J.; Ou, Y.; Han, W.; Chen, B. Hydrogenolysis Debenzylation of TADBIW in Propionic Acid and n-Butyric Acid. Chin. J. Explos. Propell. 2004, 27, 10-12.

48. Sanderson, A.J.; Warner, K.; Wardle, R.B. Process for Making 2,4,6,8,10,12-Hexanitro-2,4,6,8,10,12-Hexaazatetracyclo [5.5.0.05,903,11] Dodecane. US Patent US 6,391,130 B1, 15 February 2000.

49. Bayat, Y.; Mokhtari, J.; Farhadian, N.; Bayat, M. Heteropolyacids: An Efficient Catalyst for Synthesis of CL-20. J. Energ. Mater. 2012, 30, 124-134. [CrossRef]

50. Surapaneni, R.; Damavarapu, R. Process improvements in CL-20 manufacture. In Proceedings of the 31st International Annual Conference "ICT on Energetic Materials", Karlsruhe, Germany, 27-30 June 2000; pp. 108/1-108/4.

51. Chen, S.; Qiu, W.; Yu, Y. Oxidation Debenzylation and Acetylation of Hexabenzylhexaazaisowurtzitane. Chin. J. Explos. Propell. 2000, 23, 11-12.

52. Qiu, W.; Chen, S.; Yu, Y. The Structure of 2,4,8,10-tetrabenzyl-6,12-dibenzoyl-2,4,6,8,10,12-hexaazatetracyclo [5.5.0.05,9.03,11] dodecane. Chin. J. Explos. Propell. 2001, 24, 62-63.

53. Pang, S.; Yu, Y.; Zhao, X. Separation and Identification of the Nitrosation Products from Monoacetyltribenzoyldibenzylhexaazaisowutzitane. Chin. J. Energ. Mater. 2002, 10, 1-3.

54. Pang, S.; Yu, Y.; Zhao, X. Phase Transfer Catalyze the Oxidation of Hexabenzylhexaazaisowutzitane. Acta Armamentarii 2002, 23, 276-278.

55. Pang, S.; Yu, Y.; Zhao, X. Nitrosation of the Oxidation Products of Hexabenzylhexaazaisowurtzitane. Chin. J. Explos. Propell. 2002, 25, 27-28

56. Liu, J.; Chen, S.; Yu, T.; Zhao, X. Study on Debenzylation of TADBIW by Oxidation. Chin. J. Energ. Mater. 2002, 10, 145-147.

57. Liu, J.; Chen, S.; Yu, Y.; Zhao, X. Nitrosation of the Oxidation Products of Hexabenzylhexaazaisowurtzitane. Chin. J. Explos. Propell. 2003, 26, 60-61.

58. Liu, J.; Chen, S.; Yu, Y.; Zhao, X. Oxidation of Derivatives of Hexaazaisowurtzitane. Chin. J. Explos. Propell. 2003, 26, 5-7.

59. Pang, S.; Yu, Y.; Zhao, X. Nitration of Tetraacetyldibenzylhexaazaisowutzitane with Phase Transfer Catalysts. Chin. J. Energ. Mater. 2003, 11, 222-223.

60. Pang, S.; Yu, Y. The Hydrolysis-nitration of Hexabenzylhexaazaisowurtzitane's Oxidation Products. Chin. J. Energ. Mater. 2004, 12, 41-43.

61. Pang, S.; Yu, Y.; Zhao, X. The Synthesis of Hexanitrohexaazaisowutzitane by Oxidation. Chin. J. Explos. Propell. 2004, 27, 9-11.

62. Pang, S.; Yu, Y.; Zhao, X. A Novel Synthetic Route to Hexanitrohexaazaisowurtzitane. Propell. Explos. Pyrot. 2005, 30, 442-444. [CrossRef]

63. Gore, G.M.; Sivabalan, R.; Nair, U.N.; Saikia, A.; Venugopalan, S.; Gandhe, B.R. Synthesis of CL-20: By oxidative debenzylation with cerium (IV) ammonium nitrate (CAN). Indian J. Chem. 2007, 46B, 505-508. [CrossRef]

64. Sysolyatin, S.V.; Lobanova, A.A.; Chernikova, Y.T.; Sakovich, G.V. Methods of synthesis and properties of hexanitrohexaazaisowurtzitane. Russ. Chem. Rev. 2005, 74, 757-764. [CrossRef]

65. Cagnon, G.; Eck, G.; Herve, G.; Jacob, G. Process for the 2-Stage Synthesis of Hexanitrohexaazaisowurtzitane Starting from a Primary Amine. US Patent US 7,279,572 B2, 9 October 2007.

66. Chapman, R.D.; Hollins, R.A. Benzylamine-Free, Heavy-Metal-Free Synthesis of CL-20 via Hexa(1-propenyl)hexaazaisowurtzitane. J. Energ. Mater. 2008, 26, 246-273. [CrossRef]

67. Chapman, R.D. Processes for Preparing Eartain Hexaazaisowurtzitanes and Their Use in Preparing Hexanitrohexaazaisowurtzitane. US Patent US 8,268,993 B1, 18 September 2012.

68. Li, X.; Sun, C.; Zhao, X.; Song, J. Synthesis of Hexaallylhexaazaisowurtzitane. Chin. J. Energ. Mater. 2007, 15, 490-491.

69. Dong, K.; Wang, Y.; Gong, X.; Zhang, J.; Sun, C.; Pang, S. Formyl azido substituted nitro hexaazaisowurtzitane-Synthesis, characterization and energetic properties. New J. Chem. 2013, 37, 3685-3691. [CrossRef]

70. Hou, A. The Synthesis and Its Technology of the Hexanitrohexanzaisowurtzitane; Southeast University: Nanjing, China, 2007.

71. Han, W.; Ou, Y.; Chen, B. Studies of Different Supported Palladium Catalysts for Debenzylation of the Caged Tertiary Amine. Chin. J. Fine Chem. 2004, 21, 819-822.

72. Chen, H.; Chen, S.; Hu, F.; Li, L.; Yang, R. Optical Spectral Verification of a Harmful Impurity to Palladium Catalyst. Chin. J. Spectrosc. Spect. Anal. 2007, 27, 469-472.

73. Chen, H.; Chen, S.; Li, L.; Shi, Y.; Yang, R.; Xiang, Y. Identification and Forming Process of Main Impurity in HBIW. Acta Armamentarii 2007, 28, 1179-1182.

74. Fotouhi-Far, F.; Bashiri, H.; Hamadanian, M. Study of Deactivation of Pd(OH)(2)/C Catalyst in Reductive Debenzylation of Hexabenzylhexaazaisowurtzitane. Propell. Explos. Pyrot. 2017, 42, 213-219. [CrossRef] 